\title{
Article \\ Ectopic Expression of OLEOSIN 1 and Inactivation of GBSS1 Have a Synergistic Effect on Oil Accumulation in Plant Leaves
}

\author{
Zhiyang Zhai * ${ }^{\mathbb{D}}$, Hui Liu and John Shanklin* \\ Biology Department, Brookhaven National Laboratory, BNL 463, 50 Bell Ave., Upton, NY 11953, USA; \\ huiliu1@bnl.gov \\ * Correspondence: zzhai@bnl.gov (Z.Z.); shanklin@bnl.gov (J.S.); \\ Tel.: +1-631-344-5360 (Z.Z.); +1-631-344-3414 (J.S.)
}

Citation: Zhai, Z.; Liu, H.; Shanklin, J. Ectopic Expression of OLEOSIN 1 and Inactivation of GBSS1 Have a Synergistic Effect on Oil Accumulation in Plant Leaves. Plants 2021, 10, 513. https://doi.org/10.3390/plants 10030513

Academic Editor: José Manuel Martínez-Rivas

Received: 16 February 2021

Accepted: 6 March 2021

Published: 9 March 2021

Publisher's Note: MDPI stays neutral with regard to jurisdictional claims in published maps and institutional affiliations.

Copyright: (c) 2021 by the authors. Licensee MDPI, Basel, Switzerland. This article is an open access article distributed under the terms and conditions of the Creative Commons Attribution (CC BY) license (https:// creativecommons.org/licenses/by/ $4.0 /)$.

\begin{abstract}
During the transformation of wild-type (WT) Arabidopsis thaliana, a T-DNA containing OLEOSIN-GFP (OLE1-GFP) was inserted by happenstance within the GBSS1 gene, resulting in significant reduction in amylose and increase in leaf oil content in the transgenic line (OG). The synergistic effect on oil accumulation of combining gbss1 with the expression of OLE1-GFP was confirmed by transforming an independent $g b s s 1$ mutant (GABI_914G01) with OLE1-GFP. The resulting OLE1GFP/gbss1 transgenic lines showed higher leaf oil content than the individual OLE1-GFP/WT or single gbss1 mutant lines. Further stacking of the lipogenic factors WRINKLED1, Diacylglycerol O-Acyltransferase (DGAT1), and Cys-OLEOSIN1 (an engineered sesame OLEOSIN1) in OG significantly elevated its oil content in mature leaves to $2.3 \%$ of dry weight, which is 15 times higher than that in WT Arabidopsis. Inducible expression of the same lipogenic factors was shown to be an effective strategy for triacylglycerol (TAG) accumulation without incurring growth, development, and yield penalties.
\end{abstract}

Keywords: triacylglycerol; fatty acids; oleosin; granule bound starch synthase; metabolic engineering

\section{Introduction}

The oil triacylglycerol (TAG) is an energy-dense molecule that is essential for human nutrition and constitutes feedstocks for the production of biofuels and bioproducts [1]. TAGs consist of three fatty acids esterified to a single molecule of glycerol. Photosynthetically derived sugars provide carbon skeletons, energy, and reductant for fatty acid (FA) and TAG synthesis. In plants, multiple enzymes (including acetyl-CoA carboxylase, fatty acid synthase, acyltransferases, etc.) work in concert to catalyze de novo fatty acid synthesis and TAG assembly [2,3]. TAG mainly accumulates in plant seeds. Vegetative tissues, which constitute a major portion of plant biomass, have very low levels (typically less than $0.1 \%$ of dry weight) of TAG. Recent efforts in genetic and metabolic engineering have resulted in the successful accumulation of TAG in the leaves of both dicot and monocot crop plants $[4,5]$. For example, the Push-Pull-Protect strategy resulted in the accumulation of significant levels of TAG in tobacco leaves [6] by ectopic co-expression of WRINKLED1 (encoding WRI1, a master transcriptional regulator of glycolysis and fatty acid synthesis [7,8]), DGAT1 (encoding diacylglycerol acyltransferase 1, which catalyzes the final step of TAG biosynthesis [9]), and OLEOSIN1 (OLE1, encoding a structural oil body protein [10]), in which WRI1 is for pushing, DGAT1 is for pulling, and OLE1 is for protecting oil. Reducing fatty acid (or TAG) degradation and diverting carbon from starch synthesis also favors oil accumulation. In Arabidopsis, it was found that overexpression of WRI1 alone resulted in a 2.6-2.8-fold increase in TAG accumulation in leaves. Suppression of the small subunit of Adenosine diphosphate (ADP)-glucose pyrophosphorylase (ADG1), which catalyzes the first committed step of starch synthesis, by RNAi in wild type (WT) only slightly increased TAG accumulation. However, the combination of WRI overexpression along with ADG1 RNAi produced 5.8 times more TAG than WT [11]. WRI1, DGAT1, and OLE1 overexpression with 
simultaneous silencing of $A D G 1$ and $P X A 1$, an $A B C$ transporter-like protein required for fatty acid $\beta$-oxidation in peroxisome [12], produced a transgenic sugarcane that generated 95 times more TAG in leaves than WT [13]. Mutation of SDP1 encoding a TAG lipase also significantly boosts vegetative oil accumulation when WRI1 and DGAT1 are co-expressed in Arabidopsis [14]. Another successful strategy for promoting vegetative oil production is to change sugar partitioning. SUC2 encodes a membrane sucrose-proton symporter that is essential for sucrose phloem loading and long-distance transport [15]. The suc2 adg1 double mutant was shown to accumulate an 80-fold higher level of soluble sugars (sucrose, glucose, and fructose combined) in leaves compared to WT. Correspondingly, the high sugar content in suc2 adg1 boosted TAG accumulation to more than $1 \%$ of dry weight (DW) [16].

Starch is the major storage carbohydrate in plants. It is accumulated at high levels as granules in storage tissues such as grains, tubers, and roots, and deposited in most vegetative tissues to a lesser extent. The dominant components of starch granules are two types of glucan polymer: amylose (making up 30\%) and amylopectin (which constitutes $\sim 70 \%$ ). Lipids represent the most important minor component associated with the starch granules $[17,18]$. The lipids associated with starch granules can be surface lipids or internal lipids. TAG is a major component of surface lipids in maize and wheat starch granules. Other surface lipids including glycolipids and phospholipids derive from the amyloplast membrane [19]. In contrast, internal lipids are mainly composed of monoacyl lipids (free fatty acid and lysophospholipids) in amounts that are proportional to the amylose content [20]. The starch biosynthesis pathway is well established, involving ADP-glucose pyrophosphorylase, starch synthase (SS), and starch branching enzyme (SBE). ADP-glucose, which is synthesized from glucose-1-phosphate and ATP in a reaction catalyzed by ADPglucose pyrophosphorylase, is the precursor for both amylose and amylopectin. In the remaining steps of starch synthesis, SS catalyzes the synthesis of an $\alpha(1 \rightarrow 4)$ linkage. The $\alpha(1 \rightarrow 6)$ branches in starch polymers are brought by SBE. Two different types of SS are present in plants: granule-bound SS (GBSS) and soluble SSs. GBSS is responsible for amylose biosynthesis, while soluble SSs mainly synthesize amylopectin $[17,18]$.

In the current research, characterization of a high-leaf-oil OLE1-GFP transgenic line (named OG hereafter) showed that T-DNA inserted within the GBSS1 gene disrupted the only granule-bound starch synthase gene in Arabidopsis. Overexpression of OLE1-GFP in an independent gbss1 mutant resulted in significantly higher leaf TAG content than its expression in WT or gbss1 without expression of further transgenes. Unlike OLE1-GFP/WT transgenic plants, which showed growth defects, $O G$ plants are visibly indistinguishable from $\mathrm{WT}$, making it a strong candidate line for further engineering of vegetative oil. Stacking of WRINKLED1, DGAT1, and Cys-OLE1 (encoding an engineered sesame OLE1 [21]) in OG further increased leaf TAG content to $2.26 \%$ of DW, demonstrating that deficiency of GBSS1 is compatible with commonly employed Push-Pull-Protect strategies [6] for boosting vegetative oil accumulation.

\section{Results}

2.1. An Arabidopsis OLE1-GFP Transgenic Line with a T-DNA Insertion in GBSS1 Accumulated Significant Leaf TAG

Here we characterize a previously described Arabidopsis OLE1-GFP transgenic line called OG [22]. The transgenic line was generated by transforming Arabidopsis WT (WT) plant with the binary vector pGKPGWG containing the OLE1 genomic sequence with an in-frame GFP C-terminal extension under the control of the CaMV35S promoter. While the growth of $O G$ is similar to that of WT plants, its mature leaves showed strong GFP fluorescence signal and accumulated over 4 times more TAG than WT plants (Figure 1A-C), Winichayakul et al. had shown little if any TAG increase in WT Arabidopsis leaves overexpressing sesame OLE [21]. In other work, Arabidopsis plants expressing CaMV35S:OLE1GFP did not accumulate significant amounts of fusion proteins in the leaves either [23]. Other WT OLE1-GFP transgenics transformed with the same construct as OG have shorter siliques and lower GFP fluorescence signal relative to OG (Supplementary Figure S1). 
Transient expression of the same construct was also conducted in Nicotiana benthamiana ( $N$. benthamiana) leaves. Three independent experiments showed that expression of Ole1-GFP increased the leaf TAG content by 1.4-, 1.6- and 2.3-fold compared with empty vector controls (Supplementary Figure S2). To explain these apparently conflicting results, we hypothesized that the T-DNA insertion site of $O G$ might be contributing to the observed high leaf TAG in OG. Crosses with WT OG $\times$ Col0 resulted in F2 seeds with a 3:1 resistant:susceptible Kanamycin phenotype indicating that $O G$ contains a single T-DNA locus of insertion. Thermal asymmetric interlaced PCR (TAIL-PCR) was utilized to identify the sequence flanking the OG T-DNA insertion site. At the borders of the T-DNA insert site we identified a sequence encoding the 10th exon of GBSS1 (Figure 1D). Genotyping further confirmed GBSS1 was disrupted in OG by using PCR primers designed against either side of insertion and the left border of the T-DNA construct (Figure 1E). Quantification of expression of GBSS1 in leaves of OG showed that its expression was reduced by more than $90 \%$ compared to WT (Figure 1F). GBSS1 is the only starch synthase capable of catalyzing the synthesis of amylose in starch granules of the chloroplast. Iodine staining of $O G$ leaves was similar to that of an independent gbss1 mutant (GABI_914G01 [24]), confirming that most if not all of the amylose was depleted and that the function encoded by GBSS1 in OG was indeed compromised by the T-DNA insertion (Figure 1G). In sum, we found OG had a unique genetic background resulting from the insertion of the OLE 1-GFP overexpression T-DNA in the GBSS1 gene, thereby creating a GBSS1-deficient background.

A

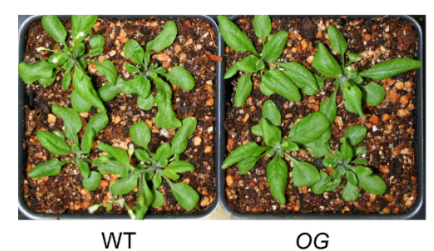

D

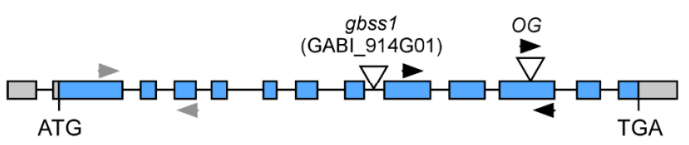

E

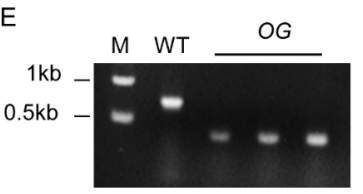

B

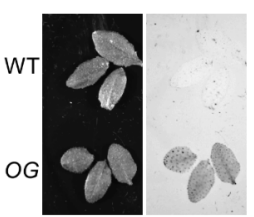

C

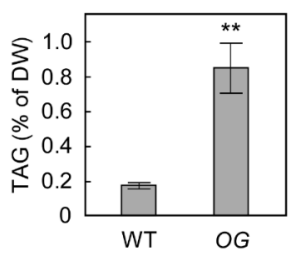

G
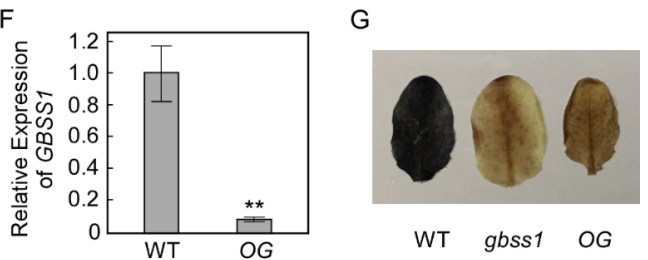

Figure 1. Characterization of an Arabidopsis OLE1-GFP transgenic line (OG). (A) Phenotype of 1month-old wild type (WT) and OG plants. (B) Bright light (Left) and GFP fluorescence (Right) of WT and OG by fluorescence image analyzer (ImageQuant LAS4000). (C) Leaf TAG contents of WT plants (WT) and OG. Values are means $\pm \mathrm{SE}(n=6)$ of measurements on mature leaves of 1-month-old soil grown plants. Asterisks denote statistically significant difference from WT (Student's $t$ test, ${ }^{* *}, p<0.01$ ). (D) Schematic illustration of the exon-intron structure of the GBSS1 gene. Exons are represented by blue boxes. Grey boxes represent the $5^{\prime}$ and $3^{\prime}$ UTRs. Translation start (ATG) and stop (TAG) codons are indicated. Open inverted triangles indicate T-DNA insertion sites for OG and gbss1 mutant respectively. Black arrowheads indicate primers for genotyping OG. Grey arrowheads represent primers used in quantitative real time PCR (qRT-PCR) for quantifying gene expression of GBSS1. (E) Genotyping of OG with primers in (D). M is DNA marker. (F) Analysis of GBSS1 expression in $\mathrm{WT}$ and $O G$ plants. The values are means \pm SE of measurements taken by qRT-PCR on three individual plants employing primers (gray arrow) shown in (D). F-box expression was used as a control for normalization $(* *, p<0.01)$. (G) Iodine staining of leaves starch granules isolated from $0.5 \mathrm{~g}(\mathrm{FW})$ leaves of WT and $O G$ plants. 


\subsection{Overexpression of OLE1 in a gbss1 Background Resulted in Higher TAG Accumulation Than} in WT

Increased accumulation of TAG in OG suggested that deficiency of GBSS1 together with ectopic expression of OLE1 may have synergistic roles in increasing leaf TAG synthesis. To test this hypothesis, an independent gbss1 mutant (GABI_914G01 [24], Figure 1B) (and WT as a control) were transformed with OLE1-GFP driven by the CaMV35S promoter. More than 10 independent transgenic lines for both genetic backgrounds were identified. Three randomly selected transgenic lines for each background were taken to the T3 generation to identify homozygous lines. TAG quantification in mature leaves of 4-week-old plants showed that while the TAG content of gbss1 was no different from WT, TAG in OLEGFP/gbss1 was significantly higher than either that of OLE1-GFP/WT or the gbss1 mutant (Figure 2). These data support the hypothesis that the effects of $g b s s 1$ and overexpression of OLE1 are synergistic with respect to leaf TAG accumulation.

Stacking of lipogenic factors WRINKLED1, DGAT1, and Cys-OLE1 into OG significantly increased leaf TAG accumulation.

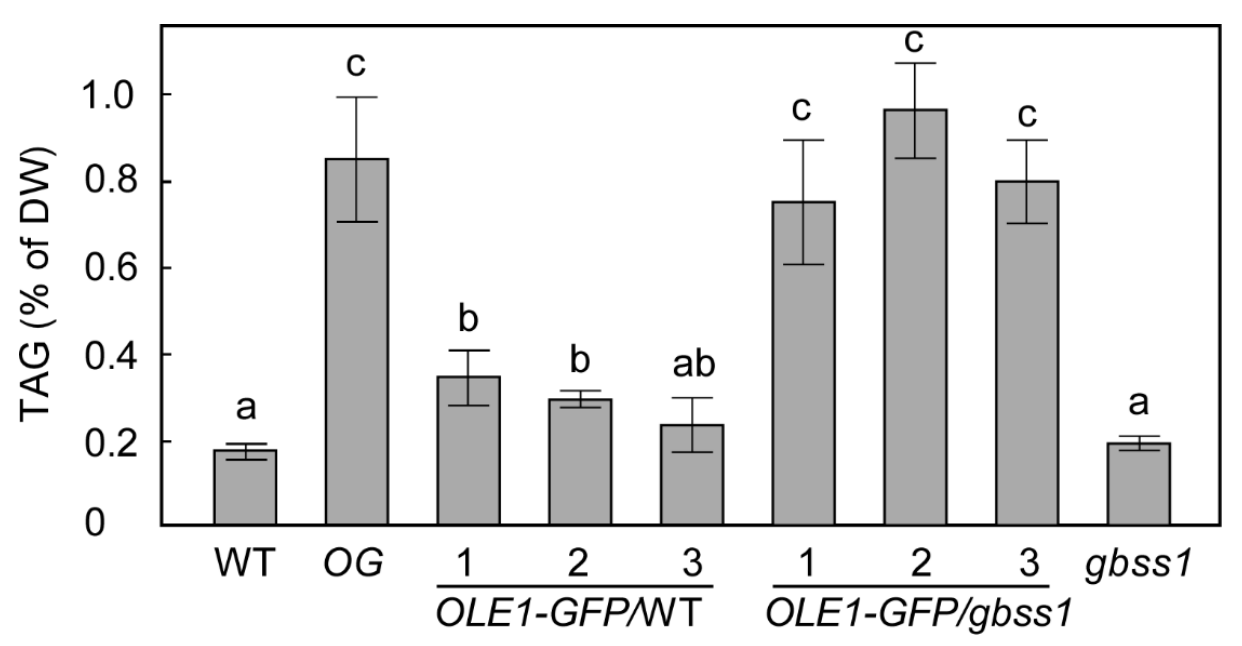

Figure 2. Overexpression of OLE1-GFP and gbss1 mutation have synergistic roles in increasing TAG content in leaves. TAG quantification in leaves of varied genotypes as indicated. Values are means \pm SE of measurements on mature leaves of 1-month-old soil grown plants. Levels indicated with different letters above histogram bars are significantly different (Student's $t$ test for all pairs of genotypes, $n=6, p<0.05)$.

Vegetative oil accumulation often has a negative impact on plant growth. However, OG grows indistinguishably from WT (Figure 3), which makes it suitable for further genetic manipulation to hyper-accumulate TAG in leaves.

To increase leaf TAG accumulation, three genes encoding three lipogenic factors, CysOLE1, WRI1, and DGAT1, were assembled into a single T-DNA (Figure 3A). Based on transient expression assays in N. benthamiana, in which WRI1 was found to be more potent as an N-terminal fusion with cyan fluorescent protein (CFP) (Supplementary Figure S3), CFP-WRI was incorporated into the T-DNA construct. To monitor the levels of Cys-OLE1, GFP was fused in frame at its $C$ terminus. The resulting Cys-OLE1-GFP, CFP-WRI1, and DGAT1 construct, referred as OWD hereafter, (Figure 3A) was validated by transient expression in N. benthamiana leaves. Both WRI1 and Cys-OLE1 exhibited strong expression 2 days after infiltration as monitored by fluorescence signal localization in the nucleus and oil bodies, respectively (Supplementary Figure S4A). At 4 days after infiltration, TAG accumulation of OWD-infiltrated leaves reached 3.3\% (DW), which corresponds to a 60fold increase compared with empty vector controls (Supplementary Figure S4B). After validation, OWD was transformed into the Arabidopsis WT (WT) and OG backgrounds. For each transformation, 10 independent transformants were screened for elevated TAG 
in the T2 generation, and 3 homozygous transgenic lines with highest TAG levels were advanced to the T3 generation. Ectopic overexpression of OWD was detrimental to growth, but more so in the OG background than in WT (Figure 3B). We also observed that OWD transgenic plants showed some reduced growth, delayed flowering, and reduced fecundity (data not shown). TAG accumulation in leaves of OG OWD transgenic plants were $1.64 \%$ on dry weight basis vs. $0.6 \%$ in WT leaves of OWD transgenics (Figure 3C).

A
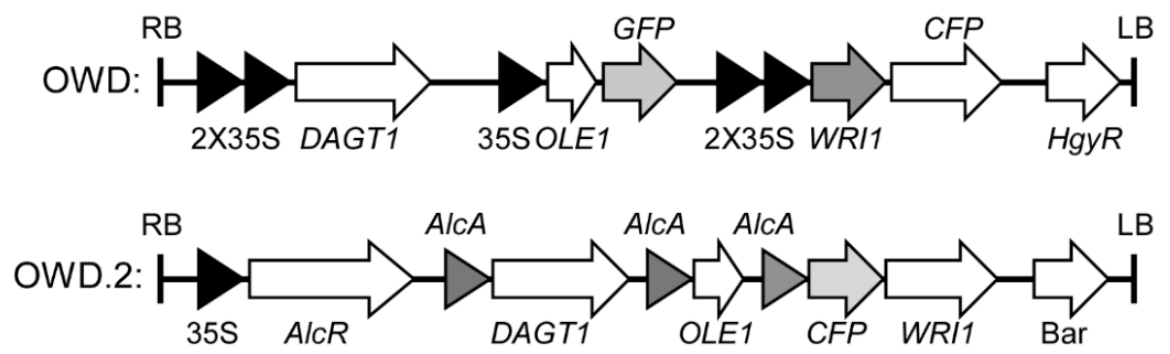

B

$\begin{array}{lllll}\text { B WT } & \text { OWD/OG } 1 & \text { OWD/WT } 1 & \text { OWD.2/OG } 1 & \text { OWD.2/WT } 1\end{array}$

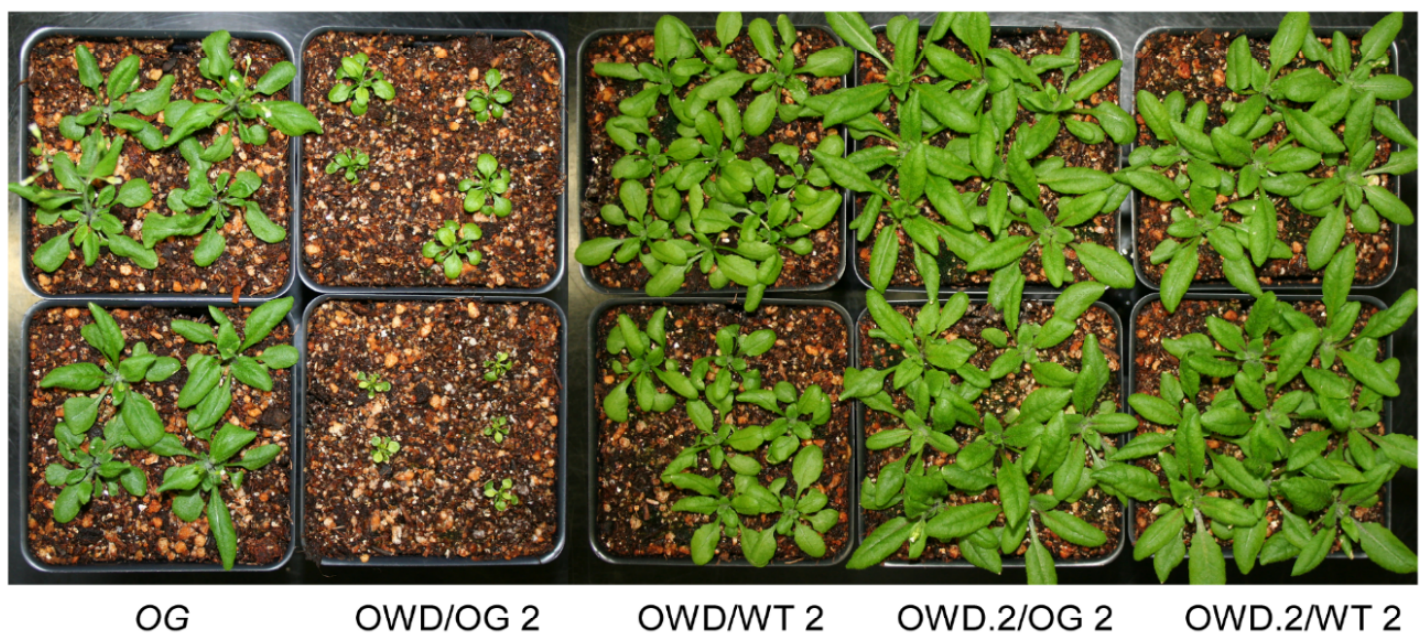

C

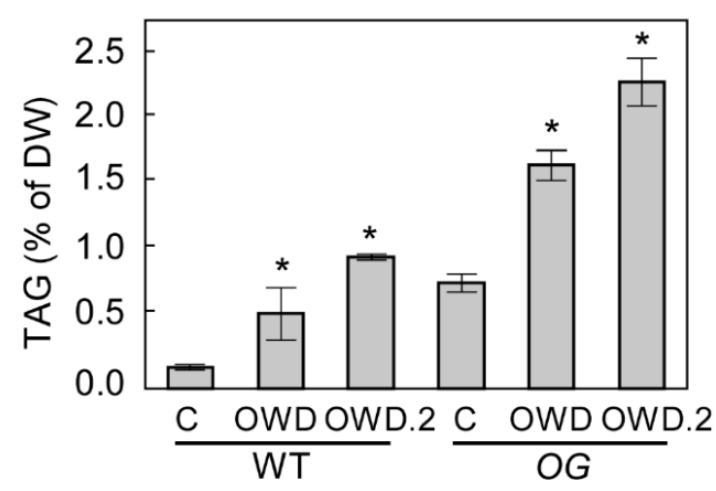

Figure 3. Stacking WRI1 and DGAT1 and Cys-OLE1 in GO significantly boosts its leaves oil content. (A) Schematic illustration of T-DNA construct designed for constitutive or ethanol inducible expression of Cys-OLE, WRI1, and DGAT1 (OWD and OWD.2). (B) Representative phenotype of constitutive or inducible OWD transgenic plants. Two representative 8-week-old soil grown transgenic lines for constitutive (OWD) or inducible expression (OWD.2) of OWD in WT or GO background were shown. (C) Leaves TAG quantification for varied genotypes in (B). $\mathrm{C}$ is a non-transgenic plant. Values are means \pm SE $(n=6)$. For the OWD.2 plant, TAG was measured after 5 days of induction by irrigating with $2 \%$ of ethanol solution. Asterisks $\left({ }^{*}\right)$ denote statistically significant differences compared with $C$ (Student's $t$ test, $p<0.01$ ). 
To further explore the feasibility of this strategy in boosting vegetative TAG, we created a construct in which WRI1 was placed under the control of ethanol-inducible promoter, and OLE1 and DGAT1 were under strong constitutive control. As before, the ethanol-inducible TAG construct was first validated by transient expression in N. benthamiana. One day after agroinfiltration, the expression of WRI1 was induced by drenching roots with $1 \%$ or $2 \%$ of ethanol. Four days of ethanol induction elevated TAG levels 15-fold, whereas without ethanol induction, AlcA:WRI1-infiltrated leaves barely accumulated any TAG. Interestingly, incubation of roots in $2 \%$ ethanol resulted in similar leaf TAG accumulation to that resulting from 4 days of agroinfiltration with 35S:WRI1 (Supplementary Figure S5). We next constructed OWD.2, in which each of the three genes were placed under the control of the AlcA promoter (Figure 3A) and transformed it into the Arabidopsis WT and OG backgrounds, respectively. As expected, both WT and OG OWD.2 transgenic plants did not show any abnormalities during development in the absence of ethanol (Figure 3B). TAG accumulation in the leaves of 8-week-old WT and OG OWD.2 transgenic plants reached $1 \%$ and $2.26 \%$, respectively, after 5 days induction with $2 \%$ of ethanol solution vs. $0.6 \%$ and $1.64 \%$ TAG in constitutive expression of OWD in WT and OG (Figure 3C), providing support for inducible expression as a viable strategy for increasing vegetative TAG accumulation.

\section{Discussion}

Here, we show that an Arabidopsis WT OLE1-GFP transgenic line (OG) [22] contains a single T-DNA insertion within a starch synthase gene, GBSS1, which results in a significant reduction of amylose content. The elevated leaf TAG phenotype characteristic of $O G$ results from the serendipitous disruption of GBSS1 by the OLE1-GFP T-DNA. This is supported by phenocopying the elevated leaf TAG of OG by combining the overexpressing OLE1-GFP in a gbss1 background (GABI_914G01), which is higher than either OLE1-GFP/WT or the gbss1 mutant alone.

As the major non-structural carbohydrate in plants, starch serves as an important transient form of carbon storage that fuels plant metabolism and growth in the absence of photosynthesis. Starch is almost entirely made up of amylose and amylopectin [25]. GBSS1 is the starch synthase that catalyzes synthesis of amylose in chloroplast starch granules. TAG synthesis and starch synthesis compete for carbon flux [26], so the overexpression of OLE1-GFP in a gbss1 mutant background results in higher TAG accumulation most likely because of reduced competition for carbon. An alternative possibility is that monoacyl lipids (non-esterified fatty acid and lysophospholipids) that under normal conditions strongly associate with amylose could be channeled towards TAG synthesis in the gbss1 mutant. However, TAG content in the gbss1 mutant is no different from that of WT, indicating that the reduction of amylose alone is not sufficient to increase TAG accumulation.

Mutations such as $s d p 1$, which is deficient in a triacylglycerol lipase, and $\operatorname{tg} d 1$, which is a leaky mutant for TRIGALACTOSYLDIACYLGLYCEROL1 (TGD1) in which approximately half of its seeds abort, resulting in high-leaf-TAG phenotypes, require sugar supplementation for germination on Murashige and Skoog (MS) medium [14,27]. However, OG, which also shows elevated vegetative TAG levels, grows from seed to seed indistinguishably from WT (Figure 3), making it a useful host for further increases in vegetative TAG accumulation. Cys-OLE1, WRI1, and DGAT1 (OWD) were chosen for ectopic co-expression in $O G$ because each of these genes have been shown to increase oil accumulation in vegetative tissues, and their co-expression has been shown to synergistically boost TAG accumulation $[6,8,11,23,28-30]$. In this study, transient co-expression of OWD in tobacco leaves increased TAG levels 60-fold relative to empty vector controls (Supplementary Figure S4), confirming our gene combination is effective in boosting oil in vegetative tissues.

Although constitutive co-expression of OWD in both Arabidopsis WT and OG plants further elevated TAG level to $0.6 \%$ and $1.64 \%$, respectively, severely adverse effects on growth were observed (Figure 3 ). It was previously shown that AGPRNAi-WRI1 overex- 
pression delayed the development and expansion of cotyledons and leaves [11]. In the present study, ectopic overexpression of OLE1-GFP alone was also found to negatively affect silique development and corresponding seeds yields (Supplementary Figure S1). To circumvent these negative effects of constitutive overexpression of OLE1 and WRI1 on growth, ethanol-inducible expression of OWD was tested. Ethanol inducible gene expression in plants has many advantages when compared with other inducible systems, such as dexamethasone or heat shock, in minimizing negative impacts while maximizing induction sensitivity [31]. The OWD.2 T-DNA construct that placed all three genes under the control of the AlcA promoter was introduced into Arabidopsis WT and OG plants using floral dip transformation. As expected, both WT and OG OWD.2 transgenic plants established and grew similarly to WT transformants (Figure 3). Five days of ethanol induction further elevated TAG content to $1 \%$ and $2.26 \%$ in WT and OG OWD. 2 transgenics, respectively, providing proof-of-concept for inducible production of oil in vegetative tissues.

\section{Materials and Methods}

\subsection{Plant Materials and Growth Conditions}

Arabidopsis thaliana wild-type (Col-0), OG transgenic line [22], and gbss1 mutant (GABI_914G01 [24]) seeds were surface sterilized and selected on agar plates containing one-half-strength Murashige and Skoog salts. After 1 week, seedlings were transplanted to moist PM-15-13 AIS MIX (Lehle Seeds). All plants were grown in a $16 \mathrm{~h}$-light/ $8 \mathrm{~h}$-dark photoperiod (at a photosynthetic photon flux density of $250 \mu \mathrm{mol} \cdot \mathrm{m}^{-2} \cdot \mathrm{s}^{-1}$ ) at a $23^{\circ} / 19^{\circ} \mathrm{C}$ light/dark temperature regime and $75 \%$ relative humidity.

Thermal asymmetric interlaced PCR (TAIL-PCR) was performed as described [32] with primers as listed in Supplemental Table S1 to identify the sequence flanking of the T-DNA in OG.

\subsection{Genetic Constructs}

OLE1 (AT4G25140), WRI1 (AT3G54320), and DGAT1 (AT2G19450) were amplified by PCR from genomic DNA or seed complementary DNA of Arabidopsis by using the primer pairs listed in Table S1. The PCR products were cloned into the gateway pDONR/Zeo Vector (Invitrogen) by BP reaction and sub-cloned into the plant gateway binary vector: pGKPGWG [33], pGWB45, or pGWB414 [34] through LR reaction. Cys-OLE1 attained as a custom synthesized sequence (GenScript, Piscataway, NJ, USA) was amplified and cloned into pCHF3 between KpnI and XbaI. For GBSS1 RNAi, 207bp of its coding region was cloned into vector pRNAi-GG [35]. For assembling OWD, DAGT1 or Cys-OLE1 expression cassette containing $35 \mathrm{~S}$ promoter, gene, and terminator was amplified from the corresponding plant binary vector and sub-cloned into the PmeI and HindIII sites of pGWB45 containing WRI1 through in-fusion (Clontech), respectively. For ethanol inducible expression of three genes (OWD.2), GFP-WRI1 fragment of pMDC43 containing WRI1 was excised using $K p n \mathrm{I}$ and PacI and inserted into the corresponding sites in pBJ36_AlcA; DAGT1 and cysOLE1 were amplified by PCR, excised using KpnI and XbaI, and inserted into pBJ36_AlcA, respectively. Then, GFP-WRI1, DAGT1, or Cys-OLE1 expression cassette (each comprising AlcA promoter, gene, and terminator amplified from the corresponding $\mathrm{pBJ} 36$ vector) were one by one sub-cloned into NotI of pMBLART_AlcR by in-fusion.

RNA isolation and quantitative real time PCR ( $q R T-P C R)$ were carried out as previously described [36]. Gene-specific primers for GBSS1 and F-box (At5g15710) were used as a reference gene (Table S1).

\subsection{Preparation of Leaf Starch Granules and Iodine Staining}

Leaf starch granules were isolated according to Zeeman et al. [37].

Transient gene expression in N. bethamiana was performed as described [38]. For TAG quantification, 8 leaves from 4 plants ( 2 from each) were agroinfiltrated with the same construct combination.

TAG quantification was performed as previously described [16]. 
Supplementary Materials: The following are available online at https:/ / www.mdpi.com/2223-774 7/10/3/513/s1, Figure S1: Characterization of WT OLE1-GFP transgenic (OLE1-GFP/WT) plants from independent transformation with the same genetic construct that was described to create the OG line. (A) OLE1-GFP/WT lines showed shorter siliques than OG. Arrows point to abnormal siliques of Ole1-GFP transgenic plants. (B) GFP fluorescence signal of OLE1-GFP/WT is significantly lower than OG. Bright light (above) and GFP fluorescence (below) of WT, GO, and a representative OLE1GFP/WT line by fluorescence image analyzer (ImageQuant LAS4000); Figure S2: Oil accumulation in $N$. benthamiana leaves transiently expressing OLE1-GFP. EV is empty vector, and 1, 2, and 3 stand for three independent transient assay experiments. Values are means \pm SE of measurements on 8 leaves from 4-5-week-old N. benthamiana plants infiltrated with Agrobacterium for 4 days. Asterisks denote statistically significant differences compared with EV (Student's $t$ test, $p<0.01$ ); Figure S3: TAG levels in N. benthamiana leaves that were transiently transformed with EV (empty vector), WRI1, CFP-WRI1 (CFP fused to the $\mathrm{N}$ terminus of WRI1), or WRI1-CFP (CFP fused to C terminus of WRI1). Values in this figure are means \pm SE of measurements on 8 leaves from 4-5-week-old N. Benthamiana plants infiltrated with agrobacterium for 4 days. Asterisks denote statistically significant differences compared with WRI1 (Student's $t$ test, $p<0.01$ ); Figure S4: Laser scanning confocal images showed the coexpression of WRI1 and OLE1 in N. benthamiana epidermis cells transiently transformed with OWD (with Cys-OLE1-GFP, CFP-WRI1, and DGAT1 contained in one engineered T-DNA). Bar $=50 \mu \mathrm{m}$. Values in this figure are means $\pm \mathrm{SE}$ of measurements on 8 leaves from 4'5-week-old $N$. Benthamiana plants infiltrated with agrobacterium for 4 days; Figure S5: TAG levels in in N. benthamiana leaves that were transiently transformed with AlcA:WRI1 (ethanol inducible) or 35S:WRI1 (constitutive). Expression of WRI1 was induced by irrigating with $0 \%, 1 \%$, or $2 \%$ of ethanol solution for 4 days. Asterisks denote statistically significant differences compared with $0 \%$ of ethanol induction (Student's $t$ test, $p<0.01)$. Values in this figure are means \pm SE $(n=8)$; Table S1: Primer sequences used in this study.

Author Contributions: Z.Z. and J.S. conceived the study. Z.Z. and H.L. performed experiments. Z.Z., H.L., and J.S. analyzed data. Z.Z. and J.S. wrote the manuscript. All authors have read and agreed to the published version of the manuscript.

Funding: This research was funded by the Division of Chemical Sciences, Geosciences, and Biosciences, Office of Basic Energy Sciences, of the US Department of Energy (grant DOE KC0304000) and DOE Center for Advanced Bioenergy and Bioproducts Innovation (U.S. Department of Energy, Office of Science, Office of Biological and Environmental Research under Award Number DE-SC0018420).

Institutional Review Board Statement: Not applicable.

Informed Consent Statement: Not applicable.

Data Availability Statement: The data presented in this study are available in this article or supplemental materials here.

Acknowledgments: Identification of the insertion site of OLE1-GFP in the OG line and creation of OLE1-GFP/WT and OLE1-GFP/gbss1 lines was supported by the Division of Chemical Sciences, Geosciences, and Biosciences, Office of Basic Energy Sciences, of the US Department of Energy (grant DOE KC0304000); creation of the inducible OWD construct and phenotypic lipid analyses were funded by the DOE Center for Advanced Bioenergy and Bioproducts Innovation (U.S. Department of Energy, Office of Science, Office of Biological and Environmental Research under Award Number DE-SC0018420). Any opinions, findings, and conclusions or recommendations expressed in this publication are those of the author(s) and do not necessarily reflect the views of the U.S. Department of Energy.

Conflicts of Interest: The authors declare no conflict of interest.

\section{References}

1. Horn, P.J.; Benning, C. The plant lipidome in human and environmental health. Science 2016, 353, 1228-1232. [CrossRef] [PubMed]

2. Ohlrogge, J.; Browse, J. Lipid biosynthesis. Plant Cell 1995, 7, 957.

3. Rawsthorne, S. Carbon flux and fatty acid synthesis in plants. Progress Lipid Res. 2002, 41, 182-196. [CrossRef]

4. Zhou, X.-R.; Bhandari, S.; Johnson, B.S.; Kotapati, H.K.; Allen, D.K.; Vanhercke, T.; Bates, P.D. Reorganization of Acyl Flux through the Lipid Metabolic Network in Oil-Accumulating Tobacco Leaves. Plant Physiol. 2020, 182, 739-755. [CrossRef] [PubMed] 
5. Parajuli, S.; Kannan, B.; Karan, R.; Sanahuja, G.; Liu, H.; Garcia-Ruiz, E.; Kumar, D.; Singh, V.; Zhao, H.; Long, S.; et al. Towards oilcane: Engineering hyperaccumulation of triacylglycerol into sugarcane stems. GCB Bioenergy 2020, 12, 476-490. [CrossRef]

6. Vanhercke, T.; El Tahchy, A.; Liu, Q.; Zhou, X.R.; Shrestha, P.; Divi, U.K.; Ral, J.P.; Mansour, M.P.; Nichols, P.D.; James, C.N.; et al. Metabolic engineering of biomass for high energy density: Oilseed-like triacylglycerol yields from plant leaves. Plant Biotechnol. J. 2014, 12, 231-239. [CrossRef]

7. Focks, N.; Benning, C. wrinkled1: A novel, low-seed-oil mutant of Arabidopsis with a deficiency in the seed-specific regulation of carbohydrate metabolism. Plant Physiol. 1998, 118, 91-101. [CrossRef]

8. Cernac, A.; Benning, C. WRINKLED1 encodes an AP2/EREB domain protein involved in the control of storage compound biosynthesis in Arabidopsis. Plant J. 2004, 40, 575-585. [CrossRef]

9. Zou, J.; Wei, Y.; Jako, C.; Kumar, A.; Selvaraj, G.; Taylor, D.C. The Arabidopsis thaliana TAG1 mutant has a mutation in a diacylglycerol acyltransferase gene. Plant J. 1999, 19, 645-653. [CrossRef]

10. Siloto, R.M.; Findlay, K.; Lopez-Villalobos, A.; Yeung, E.C.; Nykiforuk, C.L.; Moloney, M.M. The accumulation of oleosins determines the size of seed oilbodies in Arabidopsis. Plant Cell 2006, 18, 1961-1974. [CrossRef]

11. Sanjaya; Durrett, T.P.; Weise, S.E.; Benning, C. Increasing the energy density of vegetative tissues by diverting carbon from starch to oil biosynthesis in transgenic Arabidopsis. Plant Biotechnol. J. 2011, 9, 874-883. [CrossRef] [PubMed]

12. Zolman, B.K.; Silva, I.D.; Bartel, B. The Arabidopsis pxa1 Mutant Is Defective in an ATP-Binding Cassette Transporter-Like Protein Required for Peroxisomal Fatty Acid $\beta$-Oxidation. Plant Physiol. 2001, 127, 1266-1278. [CrossRef]

13. Zale, J.; Jung, J.H.; Kim, J.Y.; Pathak, B.; Karan, R.; Liu, H.; Chen, X.; Wu, H.; Candreva, J.; Zhai, Z.; et al. Metabolic engineering of sugarcane to accumulate energy-dense triacylglycerols in vegetative biomass. Plant Biotechnol. J. 2016, 14, 661-669. [CrossRef] [PubMed]

14. Kelly, A.A.; van Erp, H.; Quettier, A.-L.; Shaw, E.; Menard, G.; Kurup, S.; Eastmond, P.J. The Sugar-dependent1 Lipase limits Triacylglycerol accumulation in vegetative tissues of Arabidopsis. Plant Physiol. 2013, 162, 1282-1289. [CrossRef]

15. Gottwald, J.R.; Krysan, P.J.; Young, J.C.; Evert, R.F.; Sussman, M.R. Genetic evidence for the in planta role of phloem-specific plasma membrane sucrose transporters. Proc. Nat. Acad. Sci. USA 2000, 97, 13979-13984. [CrossRef]

16. Zhai, Z.; Liu, H.; Xu, C.; Shanklin, J. Sugar Potentiation of Fatty Acid and Triacylglycerol Accumulation. Plant Physiol. 2017, 175, 696-707. [CrossRef]

17. Martin, C.; Smith, A.M. Starch biosynthesis. Plant Cell 1995, 7, 971. [PubMed]

18. Buleon, A.; Colonna, P.; Planchot, V.; Ball, S. Starch granules: Structure and biosynthesis. Int. J. Biol. Macromol. 1998, 23, 85-112. [CrossRef]

19. Galliard, T. Starch: Properties and Potential; John Wiley \& Sons Incorporated: Hoboken, NJ, USA, 1987.

20. Shewry, P.R.; Stobart, K. Seed Storage Compounds: Biosynthesis, Interactions, and Manipulation; Clarendon Press Oxford: Oxford, UK, 1993.

21. Winichayakul, S.; Scott, R.W.; Roldan, M.; Hatier, J.H.; Livingston, S.; Cookson, R.; Curran, A.C.; Roberts, N.J. In vivo packaging of triacylglycerols enhances Arabidopsis leaf biomass and energy density. Plant Physiol. 2013, 162, 626-639. [CrossRef] [PubMed]

22. Fan, J.; Yan, C.; Zhang, X.; Xu, C. Dual role for phospholipid: Diacylglycerol acyltransferase: Enhancing fatty acid synthesis and diverting fatty acids from membrane lipids to triacylglycerol in Arabidopsis leaves. Plant Cell Online 2013, 25, $3506-3518$. [CrossRef] [PubMed]

23. Li, W.; Li, L.; Sun, X.; Tang, K. An oleosin-fusion protein driven by the CaMV35S promoter is accumulated in Arabidopsis (Brassicaceae) seeds and correctly targeted to oil bodies. Genet. Mol. Res. 2012, 11, 2138-2146. [CrossRef] [PubMed]

24. Seung, D.; Soyk, S.; Coiro, M.; Maier, B.A.; Eicke, S.; Zeeman, S.C. Protein Targeting to Starch is required for localising granulebound starch synthase to starch granules and for normal amylose synthesis in Arabidopsis. PLoS Biol. 2015, 13, e1002080. [CrossRef]

25. Streb, S.; Zeeman, S.C. Starch metabolism in Arabidopsis. Am. Soc. Plant Biol. 2012, 10, e0160. [CrossRef] [PubMed]

26. Ekman, Å.; Hayden, D.M.; Dehesh, K.; Bülow, L.; Stymne, S. Carbon partitioning between oil and carbohydrates in developing oat (Avena sativa L.) seeds. J. Exp. Bot. 2008, 59, 4247-4257. [CrossRef] [PubMed]

27. Xu, C.; Fan, J.; Froehlich, J.E.; Awai, K.; Benning, C. Mutation of the TGD1 chloroplast envelope protein affects phosphatidate metabolism in Arabidopsis. Plant Cell Online 2005, 17, 3094-3110. [CrossRef]

28. Zhang, M.; Fan, J.; Taylor, D.C.; Ohlrogge, J.B. DGAT1 and PDAT1 acyltransferases have overlapping functions in Arabidopsis triacylglycerol biosynthesis and are essential for normal pollen and seed development. Plant Cell 2009, 21, 3885-3901. [CrossRef]

29. Bouvier-Nave, P.; Benveniste, P.; Noiriel, A.; Schaller, H. Expression in yeast of an acyl-CoA:diacylglycerol acyltransferase cDNA from Caenorhabditis elegans. Biochem. Soc. Trans. 2000, 28, 692-695. [CrossRef] [PubMed]

30. Vanhercke, T.; El Tahchy, A.; Shrestha, P.; Zhou, X.R.; Singh, S.P.; Petrie, J.R. Synergistic effect of WRI1 and DGAT1 coexpression on triacylglycerol biosynthesis in plants. FEBS Lett. 2013, 587, 364-369. [CrossRef]

31. Roslan, H.A.; Salter, M.G.; Wood, C.D.; White, M.R.; Croft, K.P.; Robson, F.; Coupland, G.; Doonan, J.; Laufs, P.; Tomsett, A.B. Characterization of the ethanol-inducible alc gene-expression system in Arabidopsis thaliana. Plant J. 2001, 28, 225-235. [CrossRef]

32. Liu, Y.-G.; Whittier, R.F. Thermal asymmetric interlaced PCR: Automatable amplification and sequencing of insert end fragments from P1 and YAC clones for chromosome walking. Genomics 1995, 25, 674-681. [CrossRef]

33. Zhong, S.; Lin, Z.; Fray, R.G.; Grierson, D. Improved plant transformation vectors for fluorescent protein tagging. Transgenic Res. 2008, 17, 985-989. [CrossRef] [PubMed] 
34. Nakagawa, T.; Suzuki, T.; Murata, S.; Nakamura, S.; Hino, T.; Maeo, K.; Tabata, R.; Kawai, T.; Tanaka, K.; Niwa, Y. Improved Gateway binary vectors: High-performance vectors for creation of fusion constructs in transgenic analysis of plants. Biosci. Biotechnol. Biochem. 2007, 71, 2095-2100. [CrossRef]

35. Yan, P.; Shen, W.; Gao, X.; Li, X.; Zhou, P.; Duan, J. High-throughput construction of intron-containing hairpin RNA vectors for RNAi in plants. PLoS ONE 2012, 7, e38186. [CrossRef] [PubMed]

36. Zhai, Z.; Liu, H.; Shanklin, J. Phosphorylation of WRINKLED1 by KIN10 Results in Its Proteasomal Degradation, Providing a Link between Energy Homeostasis and Lipid Biosynthesis. Plant Cell 2017, 29, 871-889. [CrossRef]

37. Zeeman, S.C.; Northrop, F.; Smith, A.M.; Rees, T. A starch-accumulating mutant of Arabidopsis thaliana deficient in a chloroplastic starch-hydrolysing enzyme. Plant J. 1998, 15, 357-365. [CrossRef] [PubMed]

38. Schütze, K.; Harter, K.; Chaban, C. Bimolecular fluorescence complementation (BiFC) to study protein-protein interactions in living plant cells. In Plant Signal Transduction; Springer: Berlin/Heidelberg, Germany, 2009; pp. 189-202. 